\title{
Two Stroke High Efficient Gate Operated Magnetic Engine: Basis for Modeling Approach
}

\author{
Manoj Gattani, Pradeepta K Sahoo, Parag Diwan \\ College of Engineering Studies \\ University of Petroleum and Energy Studies \\ Dehradun. INDIA \\ manoj.gattani@hotmail.com
}

\begin{abstract}
The present report focuses on the fundamentals of modeling of a permanent magnet based gate operated repulsive magnetic piston engine further referred to as GOPI engine, applicable to electrical and mechanical power generation in largescale system. In GOPI engine, the motion of the piston is controlled with help of a gate made up of magnetic shield material. The working of the engine depends upon magnetic and mechanical material properties of the gate like thickness of the gate, type of the shield material, distance of the gate from magnets, the pole strength of magnets and mechanism used to operate the gate. In GOPI engine the removal of complete mechanism of fuel burning process of the traditional engine gives a number of advantages like low frictional losses, reduced manufacturing cost, maintenance cost and compactness.
\end{abstract}

Key Words: Magnetic engine, GOPI engine, gate, shield material, permanent magnets

\section{INTRODUCTION}

Magnetic engine as alternate energy source can be the new hope for power industry as these engines have many benefits over exiting energy sources like possible multi fuel operation, simple design, compact in size and reduced frictional losses due to lesser number of moving parts. The magnetic engine as a prime power generation source can have many other benefits like no damage to environment and human health if it is shielded properly with magnetic shield materials; no generation of waste and no need of extra cost for power storage system. It has also many benefits over existing techniques like its running cost is very less compared to fuel based engines, nearly maintenance free, one time investment for the end users, portable, independent of climatic conditions and eco-friendly device. Generally magnetic engines can be classified into three major categories viz. electromagnetic engine, permanent magnetic engine and hybrid magnetic engine where both permanent magnet and electromagnet are used.

\section{THE GOPI ENGINE BASIS}

In GOPI engine, only repulsive force of the permanent magnets has been used to produce thrust or power stroke. For this, the gate of the magnetic shield material is introduced between the fixed magnet and the movable magnet. A fixed magnet $(\mathrm{ml})$ and other movable magnet in the form of magnetic piston (m2) are arranged in such a way that $\mathrm{m} 2$ is brought again and again near to the $\mathrm{ml}$ (after a fix interval). [1] The gate remains in closed position except the piston is at its TDC.
In ordinary piston engine, piston is powered by fuel and then it rotates the crank which is attached with it. After one complete cycle the piston is at its initial position. For continuous motion the power to the piston should be supplied at fixed interval. In the GOPI engine the fixed magnet is clamped at the place which is just above the Top Dead Center (TDC) of the piston. The movable magnet is clamped on the piston head. Now when the piston moves up and down in the piston cylinder then the $\mathrm{m} 2$ will also move up and down with the piston head. After a fix interval the $\mathrm{m} 2$ will come very near to the $\mathrm{m} 1$ where it will experience repulsive force from the $\mathrm{ml}$ and will start moving down wards. The $\mathrm{m} 2$ should experience the repulsive force only when it approaches the minimum approach distance, a magnetic shield material in form of a gate which fills the gap between the $\mathrm{m} 1$ and $\mathrm{m} 2$ is used. This gate will open when the piston just starts moving downward from TDC. The reciprocating movement of the piston is converted in to rotation motion with the help of a crank - shaft arrangement. This motion is made continuous by arranging more pistons in rows and columns.

\subsection{Driving Force}

In conventional engine, the power stroke happens because fuel burn in combustion chamber and the chemical energy of the fuel produce high thrust on the piston head which rotate the attached crank and flywheel. In GOPI engine, the power stroke produces with the help of repulsive force between the fixed magnet and the magnetic piston. Magnetic force remains there for all the time but the intensity of the field is changed with help of the gate.

The piston motion for GOPI engine can be derived from Newton's second law, avoiding complex mechanism of the crank rotation, given by the following equation.

$$
\sum F=M_{p}\left(d^{2} X / d t^{2}\right)
$$

Where $\sum \mathrm{F}$ is the net force acting on the piston head in the GOPI engine and it can be given as

$\sum \mathrm{F}=\mathrm{Fm}-\mathrm{Fa}-\mathrm{Fr}-\mathrm{Fn}$

$\mathrm{Fm}=$ Magnetic force between the $\mathrm{m} 1 \& \mathrm{~m} 2$,

$\mathrm{Fa}=$ Magnetic field blocked by the gate,

$\mathrm{Fr}=$ Frictional force between the track and piston cylinder, Fn=Reduced magnetic strength of the magnet in due course of time 
$\mathrm{Mp}=$ moving mass (piston crank assembly), $\mathrm{x}=$ position of the piston in the time frame.

There may be some more factors on which the net force on piston head depends. Theoretically the above equation for the net force satisfies the working of the engine. Now, detailed discussions of the first two individual components of the Eqn. (2) are described here.

- $F m$ is the magnetic force between two identical cylindrical solid magnets placed end to end is approximately given by the following Eqn. [2]-[3]

$$
\begin{aligned}
& \mathrm{Fm}=\left[( B _ { 0 } ^ { 2 } A ^ { 2 } ( L ^ { 2 } + R ^ { 2 } ) / \mu \pi L ^ { 2 } ) \left(\left(1 / X^{2}\right)+\left(1 /(X+2 L)^{2}\right)+\right.\right. \\
& \left.\left.2 /(X+L)^{2}\right)\right]
\end{aligned}
$$

$\mathrm{B}_{0}=$ The magnetic flux intensity very close to each pole,

$\mathrm{A}=$ The area of each pole,

$\mathrm{L}=$ The length of each magnet,

$\mathrm{R}=$ The radius of eac magnet,

$\mathrm{x}=$ The separation between the two magnets.

The Fm depends upon the magnetic properties of the permanent magnet used for the engine development. Before selecting magnets, it is very essential to understand the basic properties and type of the magnets. Several basic magnetic properties like Magnetic field intensity, magnetization and demagnetization are of critical important for the permanent magnets in a permanent magnet engine [4]-[7].

Based upon various properties of the magnetic materials It is reported by Do Hyun Kang and his team that the size and performance of high speed permanent magnet engine depends on the permanent magnet material properties [8]. The engine output, heat rise, weight and cost are a few of the characteristics which are directly influenced the selection of the engine materials. Because of cost and weight are interrelated the magnet must be selected to provide the necessary air gap magnetic field and ample coercive force to compensate for possible damaging effects while minimizing the volume of material. Permanent magnet materials come in many varieties and the four most common types for machine applications are Alnico, Ferrites, SmCo material and NdFeB material [9]-[11].

It is reported by many authors that technological advancement in the rare-earth magnets, $\mathrm{SmCo}$ and $\mathrm{NdFeB}$, in recent years, these materials have become more popular for high performance applications because of their greater power density, high coercivity and high flux densities[12]-[19].

- $\quad \mathrm{Fa}$ is the blocked magnetic field by the gate and Fag is the amount of force required to pull away the gate from its neutral mode (the gate is in closed position) to produce power stroke when the $\mathrm{m}_{2}$ (magnetic piston) approaches the TDC.

The Fa,F'a and Fag entirely depends upon the shielding nature of the gate material. The shielding of a magnetic material can be described in the terms of permeability of the material[20][21].

The Fag, the magnetic inertia force is required because the gate will virtually attach to the magnets $\mathrm{m} 1$ and $\mathrm{m} 2$ as presented in the Fig 1. This happen because when the gate is placed in between or near the magnets, the magnetic field lines coming out from the magnets may retain on it, may diverted from it or may passes through it completely or may partially blocked, retained and diverted from the gate depends upon the gate properties. In any of the situations stated above, some external force is required to pull the gate away from the neutral mode and this force is known as Fag, the attaché force between the $\mathrm{m} 1$ and gate $\& \mathrm{~m} 2$ and the gate.

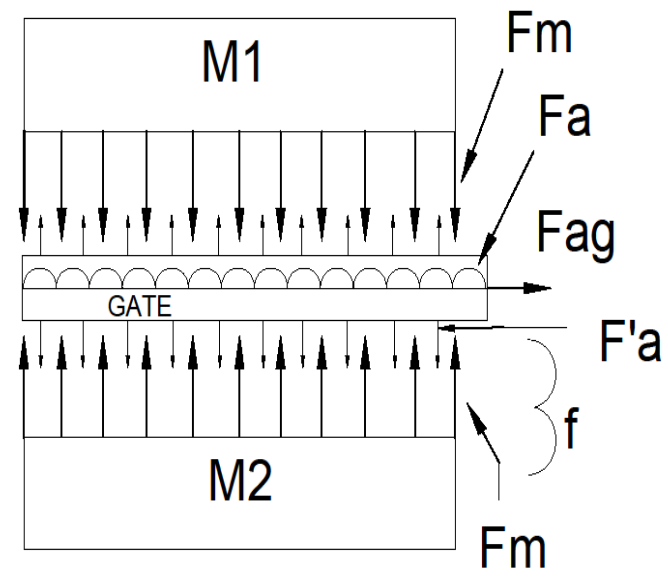

Figure 1: The Gate and the magnetic forces

The magnetic field near the gate can be understood by taking an example of a light beam passing through a material sheet where a part of light transmitted through the sheet, a part is reflected and a part of the light is absorbed by the sheet as shown in the Fig 2 . The values of light which is passes through the sheet or reflected or absorbed by the sheet can be presented in their coefficient values as coefficient of absorption(a), coefficient of reflection(r) and coefficient of transmission(t). Mathematically,

$\mathrm{a}+\mathrm{r}+\mathrm{t}=1$

To understand the working mechanism of the gate, the magnetic field very close to the gate can be treated in same manner as that of light beam near the material sheet and the gate itself as material sheet.

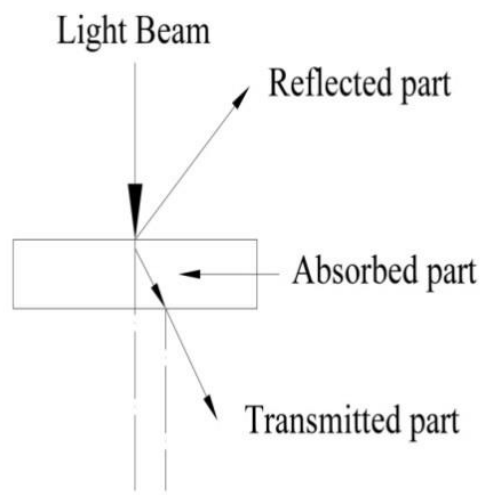

Figure 2: Light beam passes through a material sheet 
The magnetic field lines may be blocked or retained (absorbed) by the gate, or may pass (transmitted) through the gate or may get diverted (reflected) by the gate depending upon the magnetic properties of the material as shown in the Fig 3. The total value of the magnetic field near the gate can be given by the Eqn. (5)

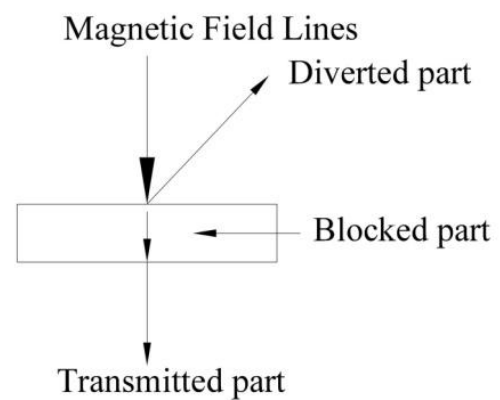

Figure 3: Magnetic field lines passes through a shield material

$\mathrm{b}+\mathrm{d}+\mathrm{p}=1$

$\mathrm{b}=$ coefficient of blocked (retaining) magnetic field by gate

$\mathrm{d}=$ coefficient of diverted magnetic field from the gate

$\mathrm{p}=$ coefficient of passing (transmitting) of magnetic field through the gate

- $\quad F r$ is the frictional force between the engine track and the magnetic piston. The magnetic piston moves on the track in a non-packed situation, though there are frictional forces between all contacts. Many other losses are also associates with magnetic engines like eddy current losses, thermal stress losses demagnetization losses [22]-[25], but for modeling of permanent magnet GOPI engine, these losses are not considered at the first stage

- $\quad F n$ is the value of magnetic strength which reduces in due course of time. Generally it is found that a magnet in stationery condition does not lose its magnetic strength in time but there is no experimental relation available between the magnetic strength reductions with continuous movement in time.

\subsection{Operating conditions}

To produce power stroke, it is most important that the gate should be in active mode and the gate cycle should be synchronized with the piston movement. For synchronization, the design of the gate plays a very important role. The design of the gate affects the power in the stroke. Mathematically it can be presented as

$\mathrm{Fm}=\mathrm{Fa}+\mathrm{F}^{\prime} \mathrm{a}$

$\mathrm{Fm}=$ magnetic force between the fixed magnet $\left(\mathrm{m}_{1}\right)$ and the magnetic piston $\left(m_{2}\right)$ (when there is no gate between the $m_{1} \&$ $\mathrm{m}_{2}$ or magnetic field lines are passing through the gate completely)

$\mathrm{Fa}=$ magnetic field blocked by the gate

F'a=Magnetic field not blocked by the gate
Now, the Fa can be expressed as

$\mathrm{Fa}=\mathrm{a} * \mathrm{Fm}$

Here, $\mathrm{a}=$ gate factor. Its value lies between 0 and 1. i.e. $0 \leq \mathrm{a} \leq 1$. The gate factor is a variable that indicates how efficiently the gate is performing i.e. how much magnetic field is diverted or blocked or passing through the gate.

The gate factor depends upon many parameters of the gate like type of gate material, thickness of material, distance of gate from the $\mathrm{ml}$ and $\mathrm{m} 2$, magnetic pole strength of the $\mathrm{ml}$ and $\mathrm{m} 2$. When the magnetic field is blocked or diverted completely by the gate, the value of the gate factor is 1 and when there is no gate in between the magnets or the magnetic field lines is passing through the gate completely as in the case when the permeability of the gate is one, the value of gate factor will be zero. In other situations its value remains between 0 and 1 . When the engine is performing efficiently, the value of gate factor will be equal to one. This time the magnetic field is either diverted or blocked completely by the gate. The value of $p$ coefficient in Eqn. (5) will become zero and the values of $b$ and $\mathrm{d}$ coefficients combined together will be one. Mathematically; When $\mathrm{a}=1 ; \mathrm{p}=0$ and $\mathrm{b}+\mathrm{d}=1$; here, $0 \leq \mathrm{b} \leq 1$ and $0 \leq \mathrm{d} \leq 1$

Further if $b=1$, the gate is blocking the magnetic field lines completely. The engine will work less efficiently as the value of Fag will be more in this situation and hence more input power is required to operate the gate. When $d=1$, the gate is diverting magnetic field lines completely. The engine will work with highest efficiency as the Fag will be equal to zero and the power required to operate the gate is minimum.

When the gate is performing with least efficiency or not working at all, the value of gate factor will be equal to or near to zero. This time the magnetic field passes through the gate completely. The value of d coefficient will be equal to zero and value of $b$ and $p$ coefficient combined will be equal to one. Mathematically

When $\mathrm{a}=0 ; \mathrm{d}=0$ and $\mathrm{b}+\mathrm{p}=1$; here, $0 \leq \mathrm{b}<1$ and $0<\mathrm{p} \leq 1$ it is to be noted that when $a=0$; the value of $b_{\max }$ will be less than one $(b<1)$ and $p_{\min }$ will be greater than zero $(0<p)$, further if the value of $b$ approaches to 1 , the gate will block maximu $m$ of the magnetic field lines but even the magnetic piston may stick to somewhere in its upward motion as some magnetic field lines will passes through the gate all the time and the nonzero value of counter force $f(f \neq 0)$ will produce hindrance in the piston movement in upward direction. The gate cycle cannot be matched with the piston movement and the engine will stop working after few cycles.

The reason behind it is that as F'm is not equal to zero, the magnetic piston cannot approaches to TDC because the repulsive force between $\mathrm{m} 1$ and $\mathrm{m} 2$ will produce hindrance in the piston movement.

Let this repulsive force between the $\mathrm{m} 1$ and $\mathrm{m} 2$ be known as counter force and represented by $f$, then the counter force can be given by

$\mathrm{f}=\mathrm{Fm}-\mathrm{Fa}$

$\mathrm{f}=\mathrm{Fm}-\mathrm{a} * \mathrm{Fm}$

$\mathrm{f}=(1-\mathrm{a}) * \mathrm{Fm}$

Two conditions under which the engine may works are described here 
Awhen there is no gate in between the magnets $\mathrm{m} 1$ and $\mathrm{m} 2$ (or magnetic field lines passes through the gate completely), then

$\mathrm{f}=(1-\mathrm{a}) * \mathrm{Fm}$

$\mathrm{f}=\mathrm{Fm}$ as a $=0$

$\mathrm{f}=\mathrm{Fm}$

This means, the counter force between the magnets $\mathrm{m} 1$ and $\mathrm{m} 2$ will be maximum and equal to Fm. In this condition, the magnetic piston will stick to somewhere in its way and the attached crank will not complete its cycle.

So for complete rotation of the crank the counterforce $f$ should be less than the magnetic force produced by the magnets of the engine at every point of the motion otherwise the engine will not work at all.

Bwhen gate block the field completely or divert the same completely i.e. $\mathrm{a}=1$, then

$\mathrm{f}=(1-\mathrm{a}) * \mathrm{Fm}$

$\mathrm{f}=(\mathrm{Fm}-\mathrm{Fm})$ as $\mathrm{a}=1$;

$\mathrm{f}=0$

This means, there will be no repulsive force or counter force between $\mathrm{m} 1 \& \mathrm{~m} 2$ when the magnetic piston is moving towards TDC. The piston will move upwards without any restriction and in this condition the speed of the piston will not be affected.

The efficiency of the engine depends upon the value of $b$ and $d$ coefficients. The efficiency of the engine may be very less if $b=1$ i.e. the gate will attach with the magnets and when $d=1$, engine will work with highest efficiency as all magnetic field lines are diverted from the gate and the value of Fag will be minimum, the power required to operate the gate will be less. Theoretical relation between the coefficients of the engine are presented in the table 1

Table 1: Theoretical relation between the GOPI engine coefficients

\begin{tabular}{|c|c|c|c|c|c|}
\hline $\begin{array}{l}\text { Sr. } \\
\text { No. }\end{array}$ & $\mathrm{b}$ & $\mathrm{p}$ & $\mathrm{A}$ & Fag & $\eta$ \\
\hline 1 & 0.0 & 1 & $=0$ & $\sim 0$ & $=0$ \\
\hline 2 & 0.25 & 0.75 & $1>>a>0$ & $>0$ & $\prod_{\eta>0} \sim$ but \\
\hline 3 & 0.5 & 0.5 & $1>a>0$ & $>>0$ & $1 \gg>\gg>0$ \\
\hline 4 & 0.75 & 0.25 & $1>a>>0$ & $\gg>0$ & $1 \gg \eta>0$ \\
\hline 5 & 1.0 & 0.0 & $a=1$ & $\mathrm{Fag}=\max$ & $1 \gg>\gg>0$ \\
\hline
\end{tabular}

In the table 1, relation between the gate coefficients, gate factor, magnetic inertia force and efficiency are presented. As it is mentioned in the Eqn. (6) and Eqn.(9) as the permeability of the gate is 1 , the engine will not work and the coefficient $p$ will have value equal to 1 . But as the permeability of the gate increases, the value of $b$ coefficient will increase and $p$ coefficient will decrease and at the same time the value of gate factor will increase.

\section{THE INPUT POWER}

To produce power stroke, it is most important that the gate should be in active mode and the gate cycle should be synchronized with the piston movement. For synchronization, the design of the gate plays a very important role. If the design of the gate is not done properly, the engine may not work. Power required to operate the gate can be supplied from some storage means of energy like battery or flywheel. For smooth working of the GOPI engine, power is required to operate the signal system to control the frequency of the engine and to operates the gate mechanism (signal systemof the GOPI engine is not described in the present paper). The total power required to operate the GOPI engine is represented as Pi and is given by $\mathrm{Pi}=\mathrm{Pgt}+\mathrm{Ps}$

$\mathrm{Pgt}=$ power required to operate the gate from its virtual attachment with $\mathrm{m} 1 \& \mathrm{~m} 2(\mathrm{~Pa})+$ power required to operate the gate from its rest i.e. neutral state (Pmi)

Pgt $=$ Pag + Pmi

Pag is the power required to operate the gate against magnetic inertia and

Pmi is the power required to operate the gate against mechanical inertia

Ps is the power required to operate the signal systemof the Gopi engine

\section{ENGINE EFFICIENCIES}

As described, efficiency of the GOPI engine depends upon many parameters like selection of permanent magnets for magnetic piston and fixed magnet, shield material for gate, operating mechanism of the gate, distance of the gate from the $\mathrm{m} 1$ and $\mathrm{m} 2$. For this research paper, a theoretical conclusion is drawn for the engine efficiency.

As the Fa, the magnetic field blocked, is a part of the net force on the piston head, the efficiency of the engine depends upon $\mathrm{Fa}$ which further depends upon the gate factor and the gate coefficients (b,d,p). So the working as well as the efficiency of the engine depends upon the basic properties of the permanent magnets and the shield material to be selected for the engine development. The gate coefficients depend upon magnetic flu $\mathrm{x}$ density (B), distance between $\mathrm{ml} \&$ gate(x1) and $\mathrm{m} 2$ \& gate $(x 2)$, permeability of the gate material $(\mu)$ thickness of the gate $(\mathrm{t})$. Mathematically, $\mathrm{b}, \mathrm{d}$ and $\mathrm{p}$ depends upon these parameters as

$b, d, p=f(B, \mu, t, x 1, x 2)$

Now the dependency of the gate factor (a) on b, d, p can be explained as

$\mathrm{a}=\mathrm{k} 1 *(\mathrm{bm} 1) *(\mathrm{dm} 2) *(\mathrm{pm} 3)$

And the magnetic inertia force, Fag as

Fag $=\mathrm{k} 2 * \mathrm{f}(\mathrm{a}, \mathrm{b}, \mathrm{d}, \mathrm{p}, \mathrm{m})$

Here, it is to be noted that when $a=0 ; \mathrm{Fag} \neq 0$ as some external force is required to pull out the gate from its neutral position. The mass of the gate (gate mechanical inertia) is presented as $m$ in the Eqn. (16). The power stroke of the engine not only depends upon the engine parameters like gate factor, gate coefficients, net force available at piston head but also on the gate geometry and the gate velocity with which the gate operates. Power in the stroke (Ps) can be explained by the Eqn . (17) as

Ps $=k 3 * f(B, \mu, x 1, x 2, t, v, g e)$ 
Here, ge is the geometry of the gate and $\mathrm{v}$ is velocity with which the gate opens. The value of the constants $\mathrm{k} 1, \mathrm{k} 2, \mathrm{k} 3, \mathrm{~m} 1, \mathrm{~m} 2$, $\mathrm{m} 3$ used in the Eqn. can be find out by conducting experiments on the GOPI engine.

It can be concluded from the table1 that the efficiency of the engine is maximu $\mathrm{m}$ when the gate coefficient $\mathrm{d}$ is equal to 1 . Its maximu $m$ value and the $b$ and $p$ coefficients equal to zero. The Fag is zero as virtual attachment between the magnets and the gate does not exist and the required input power to operate the gate is minimize. But it is also to be noted that when the gate factor is one for different values of the $b$ and $d$ coefficients, the efficiency and the Fa and Fag varies accordingly.

\section{OBSERVATION}

\section{A. Relation between input and output of the engine}

In IC engines (diesel and petrol engine), chemical energy (fuel) is changed into mechanical energy to run the output shaft. In these engines output is dependent on the input and we have to supply fuel as input to get the output. In magnetic engine, magnetic force is used to move the output shaft. In the magnetic engine, output is independent of the input as only gates and control units of the engine require input for their operation. The magnetic force is available all the time and depends only on magnetic pole strength but intensity of it at a place is changed using gate movement.

B. Magnetic engine can be a combination of engine and generator

This means, at the same time we can get mechanical and electrical output. In this design configuration, an electrical coil is wrapped round the magnetic piston cylinder. When the magnetic piston moves through the electrical coil, an electrical current will be produced. And at the same time the moving crank and flywheel will produce mechanical power at its shaft. So when we require mechanical output, we can use it as an engine or when we require electricity as output, we can use it as a generator or it can be used to produce both forms of power simultaneously.

\section{Efficiency of the engine}

The working efficiency of the GOPI engine depends upon many parameters like operating speed of the gate, frequency of the gate cycle, field blocked by the gate, operating mechanism of the gate, working efficiency of the signal system of the control units, material used for gate fabrication and virtually attachment of gate with $\mathrm{ml} \& \mathrm{~m} 2$. The input power is used to run and operate gates and control systems only. Since gates are less massive and are operating in small distance so the requirement of input power is not too much. The output power of the engine is collected at the end of the shaft.

\section{Low cost of the engine}

In GOPI engine power stroke can be attained at every 900 with four piston systems but in other engines of similar category power stroke at 900 can be attained only with more than $6 / 8$ piston systems. So fewer pistons are required in GOPI eng ine to produce same power that is produced in the RAT or Slipper based engines. At the same time solenoid cylinder can be used to increase the magnetic strength of the fixed magnet and this can produce more repulsive force between the magnets and this further can produce more torque in the power stroke.

\section{E. Simplicity}

The simplicity of the magnetic engine compared to conventional technology is one of the driving forces behind many of the recent developments. The elimination of complete mechanism of fuel burning process of the traditional engine reduces the number of parts and the complexity of the $G$ engine significantly and this potentially gives a number of advantages like low frictional losses, reduced manufacturing and maintenance cost and compactness.

\section{CONCLUSION}

It is important need of the today's era to replace oil engines or to find its substitute not because of the oil shortage but also based on environmental concerns. The engine can play a major role in the power industries and transportation sector as its running cost is very less as compared to other fuel based engines and requires no storage systems. The engine is environment friendly since it does not produce any hazardous pollutants.

Although several reports have confirmed that the magnetic engine is a viable concept, more research is required to investigate potential advantages over conventional technology. The area with most uncertainty is clearly that of piston motion control. In GOPI engine the motion of the piston is controlled with help of gate which further depends upon many parameters like thickness and type of shield material, distance of gate from magnets and mechanism used to operate the gate. A detailed study is required to investigate experimental relation between these parameters.

\section{REFERENCES}

[1] Gattani M.K. "Design and Development of Linear Magnetic Generator" International Journal of Advances Engineering and Technology, Volume 3-Issue 2, May 2012, Page 820-825.

[2] "Magnetic Fields and Forces". http://instruct.tric.edu/fgram/web/Mdipole.htm. Retrieved 200912-24.

[3] K. Bartusek, M. Cap, P. Marcon and J. Mikulka "Magnetic Susceptibility modeling using ANSYS" PIERS proceedings Marrakesh, Morocco march 20-23, 2011

[4] J M D Coey, "Magnetism and magnetic materials," Cambridge University Press

[5] S T umanski, " Handbook of magnetic measurements" e-book by CRC Press, 2011

[6] "Handbook of magnetism and advanced magnetics materials", John Wiley and Sons, 1999

[7] Jonathan E Rucker, " Design and Analysis of a permanent magnet generator for naval applications", Thesis for Master of Science, Massachusetts Institute of Technology,June 2005

[8] Do Hyun Kang, Paul Curiac, Yeon Ho Jung, Soo Jin Jung, " prospects for Magnetization of Large PM Rotors: Conclusions from a development Case Study,", , IEEE Transaction of Energy Converion, Vol 18, No 3, Sept 2003

[9] Robert C Juvinall, Kurt M Marshek, " Fundamentals of machine component design"; e-book, John Wiley \& Sons,Inc,2003

[10] M A Rahman, G R Slemon, " Promising Applications of Neodymium Iron Boron Iron Magnets in Electrical Machines," IEEE transactions on Magnetics, Vol No. 5, Sept 1985 
[11] Arnold, "Soft magnetics application guide- Basics of magnetics", The magnetic product group of SPS technology, 2003, www.arnold.com

[12] Wang Fengxiang, Zheng Wenpeng, Zong Ming, Wang Baoguo, "Design Consideration of High speed PM generators for micro turbines," IEEE Transactions, 2002

[13] Mihai Comanescu, Ali Keyhani, Min Dai, “ design and Analysis of $42 \mathrm{~V}$ Permanent Magnet Generator for automotive applications," IEEE Transaction of Energy Converson, vol 18, No. 1,2003

[14] D. Pavlik, V.K. Garg, J R Repp, J.Weiss, “A Finite Element Technique for calculating the magnet size and induction for permanent magnet machines," IEEE Transaction of Energy Converion, Vol 3, No 1, March 1988

[15] J R Hendershot, Jr \& T JE miller, "Design of brushless permanent magnet motors," Magna Physics Publishing and Clarendon Press, 1994

[16] M A Rahman, G R Slemon, " Promising Applications of Neodymium Iron Boron Iron Magnets in Electrical Machines," IEEE transactions on Magnetics, Vol No. 5, Sept 1985

[17] Edward Furlani, "Permanent magnet and electro mechanical devices", Academic Press, Elsevier,2001

[18] Karl J. Strnat, "Modern Permanent Magnets for Applications in Electro-Technology",

[19]Awaja N., Sood D. K. , Vinay T., Modeling and simulation of a flat spring for use in an electromagnetic micro generator, Smart Structures, Devices, and Systems II, Proceedings of SPIE ,2005. 5649, p.361-372. Proceedings of the IEEE, Volume 78, Number 6, June 1990, pp. 923-958

[20] Wills A P, “" On the magnetic shielding effect of cylindrical shielding, Phy,Rev9, pg 193-213

[21] Magar A,"Magnetic shields", IEEE Trans. Magn,6,1970 pg 67-75.

[22] S. M. Abu Sharkh, M R Harris, N T aghizaden Irenji, "Calculation of Rotor Eddy-Current Loss in High speed PM alternators," IEEE conference publication No 444, 1997

[23] O. Aglen, A. Andersson, " Thermal Analysis of high speed generator," IEEE Transaction 2003

[24] Unal E., Gokcen A., Kutlu Y.: Effective Electromagnetic Shielding, IEEE Microwave magazine, 1527 3342, 2006, p. 48 54," Dept. of Electric Power Engineering, FEI T U of Košice, Slovak Republic

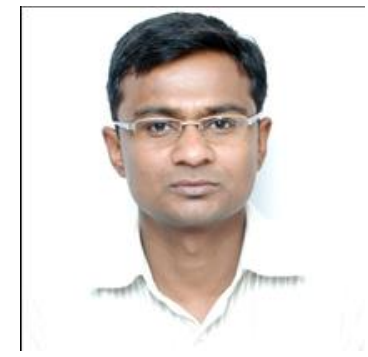

Mr. Manoj Gattani had completed his engineering and master degree from Jodhpur University (India). Currently he is working as Assistant Prof at University of Petroleum and Energy Studies, India. $\mathrm{He}$ is working on magnetic engines for power generation from last 4 years. He has five Indian patents (pending) and won several awards like young scientist award 2011, best technology of the year, 2011 etc. He is pursuing his Ph D in magnetic engine modeling and simulation from University of Petroleum and Energy Studies, Dehradun, India 